\title{
THE SINGULAR WEDGE PROBLEM IN THE NONLINEAR ELASTOSTATIC PLANE STRESS THEORY
}

$\mathrm{BY}$

\author{
ANGELO MARCELLO TARANTINO
}

Dipartimento di Scienze dell'Ingegneria, Università di Modena e Reggio Emilia, Italia

\begin{abstract}
A finite elastostatic analysis of the singular equilibrium field in the proximity of the apex of a wedge, with clamped-free radial edges and general far-field loading conditions, is performed. The problem is formulated for compressible hyperelastic sheets under a plane stress condition. An asymptotic procedure is proposed to compute the deformation and stress singular fields. Emphasis is placed on the investigation of the dependence of the order of singularity in the asymptotic Piola-Kirchhoff and Cauchy stresses on the wedge angles. The case of a half-plane bounded to a rigid substrate is studied in detail.
\end{abstract}

1. Introduction. In linear elastostatic theory, several methods of singularity analysis are available for wedge problems. Williams [1] employed the Airy stress function and separation of variables to study the single-material wedge under different boundary conditions, proposing a method of analysis that appeals in its simplicity and immediacy. Tranter [2] used the Mellin transform in conjunction with the real polar form of the Airy stress function representation of the plane elasticity solution. This method appears to be particularly suitable for asymptotic analyses of the stress field at the wedge apex (see, for example, the works of Sternberg and Koiter [3], Bogy and Sternberg [4], and Bogy [5], [6]). Green and Zerna [7] employed the complex function representation of the solution, conformally mapped the wedge into an infinite strip, and applied Fourier transforms to solve the strip problem. Hetényi [8] generated a series solutions for the wedge by overlapping known half-plane solutions. Babuska, et al. [9], used a variance of this technique to reduce the wedge problem to an integral equation. The Mellin transform was also employed by Bogy [10] to study bi-material isotropic wedges, providing conditions for a logarithmic stress singularity. For this class of problems further contributions are due to Dundurs [11], Bogy and Wang [12], Hein and Erdogan [13], Dundurs and Lee [14], Gdoutos and Theocaris [15], Theocaris and Gdoutos [16], and Rao [17]. The case of an $N$-material composite wedge was treated by Dempsey and Sinclair [18], [19]. The solution of an anisotropic wedge was proposed by Bogy [20].

Received August 1, 1996.

1991 Mathematics Subject Classification. Primary 73C50, 73M05.

(c)1999 Brown University 
In his analysis for isotropic single-material wedges subjected to extension in their plane, Williams [1] investigated the influence of three sets of boundary conditions on the radial edges: free-free, clamped-clamped, and clamped-free. Provided that the wedge angle $\phi$ (see Fig. 2) is less than $\pi$ (case of a standard wedge), he found that unbounded stresses occur at the vertex only in the case of the mixed boundary condition. On the other hand, for wedge angles between $\pi$ and $2 \pi$ (case of a reentrant wedge), all the cases considered may have stress singularities.

The preceding referenced investigations, within the scope of the classical linear theory of elasticity, typically retain the kinematic assumption of infinitesimal deformations. Nevertheless, these studies often yield strain fields that are locally unbounded and therefore in evident conflict with the underlying assumption that justifies the kinematic linearization. With reference to the specific case of crack problems, in the past two decades, some analyses have been performed to compute the singular elastostatic fields near the crack-tip by removing the requirement of infinitesimal deformations and only recently a similar analysis has been developed for the notch problem (namely, for the case of a reentrant wedge with traction-free radial edges) [21]. Within the framework of fully nonlinear elastostatics, early investigations seem to be due to Wong and Shield [22], who carried out an approximate global plane-stress analysis for an infinite neo-Hookean sheet, with a finite crack embedded and subjected to bi-axial tension at the infinite. The approximation of their approach involves the assumption (which allows the convergence of their method of the "successive substitutions") that the deformations are very large throughout the whole sheet. Such a requirement however is well-satisfied in proximity of the crack-tip only. Without any doubt, the most important paper, within the context of the plane stress theory, is that written by Knowles and Sternberg [23]. In [23], the asymptotic crack problem for a traction-free interface-crack between two dissimilar semiinfinite incompressible neo-Hookean sheets is fully explained. In particular, it is shown how the results provided by the nonlinear theory are free of oscillatory singularities of the kind predicted by the linear theory, which involve repeated interpenetration of the deformed crack-faces in vicinity of the crack-tip. Recently, the problem treated in [23] has been extended to a generalized neo-Hookean incompressible material by Geubelle and Knauss [24], [25], and [26] and to a compressible material by Tarantino [27].

The purpose of the present work is closely related to that of the aforementioned papers in nonlinear theory. Perhaps for the first time, the wedge problem is formulated and solved in a fully nonlinear context. The possibility of the wedge faces coalescing until they form an interface is admitted. The special wedge geometries of the quarter-plane, half-plane and cracked full-plane are hence included in our model. Section 2 of this paper gives the equations of the equilibrium theory of thin hyperelastic sheets under generalized plane stress conditions, whose stored energy function has the compressible neo-Hookean form. The analysis developed in Sec. 2 is then employed in Sec. 3 to formulate a local wedge problem with traction-free and clamped radial edges and general far-field loading conditions. An asymptotic analysis is carried out in Sec. 4 to compute the deformation fields near the apex of the wedge. In Sec. 5, the asymptotic Piola-Kirchhoff and Cauchy stress fields are obtained and emphasis is placed on the investigation of the dependence of the order of stress singularities or the wedge angles. In the last section, a further 
analysis is developed to study the particular case of a half-plane, in that this permits a comparison with results known in the literature.

Note. In the sequel, the Greek subscripts $\alpha, \beta$, etc. take the value 1 or 2 and bold-face letters are used to denote vectors and tensors. Differentiation with respect to a coordinate $x_{i}$ is shown by an index $i$ preceded by a comma. We also adopt the summation convention over repeated indices.

2. Nonlinear elastostatic plane stress theory. Within the framework of fully nonlinear elastostatics, the theory of plane stress for isotropic materials was developed by Adkins, Green, and Nicholas [28]. Further contributions have appeared in [29], [30] and in [31], where Yang stated the axial-symmetric case.

The theory of plane stress is based on the assumption that, for a thin plane sheet with traction-free faces and stretched by forces in its own plane (so that it remains plane after deformation), deformation and stress fields can be evaluated, with sufficient accuracy, by the deformation of the middle plane of the sheet. In other words, the solution of a three-dimensional elasticity problem is sought by reducing the original problem to a twodimensional structure which still partially depends on transversal deformation. In Love's terminology, the problem mentioned is that of generalized plane stress (cf. $§ 146$ of [32]). The theory of plane stress (contrary to that of plane strain) is of course approximated and the smaller the sheet-thickness with respect to any characteristic in-plane dimensions, the more valid it becomes.

Let $\left(x_{1}, x_{2}, x_{3}\right)$ be rectangular Cartesian material coordinates of a body that, in the undeformed configuration, occupies the closure of the regular cylindrical region $\mathcal{R}$ (of the three-dimensional Euclidean space) described by

$$
\mathcal{R}=\left\{\mathbf{x} \mid\left(x_{1}, x_{2}\right) \in \Omega \text { and }-\frac{1}{2} t<x_{3}<\frac{1}{2} t\right\},
$$

where $\Omega$ denotes the cross section of $\mathcal{R}$ in the mid-plane $x_{3}=0$ and $t$ denotes the thickness (Fig. 1).

The deformed configuration is given by the vector field

$$
\varphi: \overline{\mathcal{R}} \rightarrow \mathbb{R}^{3}
$$

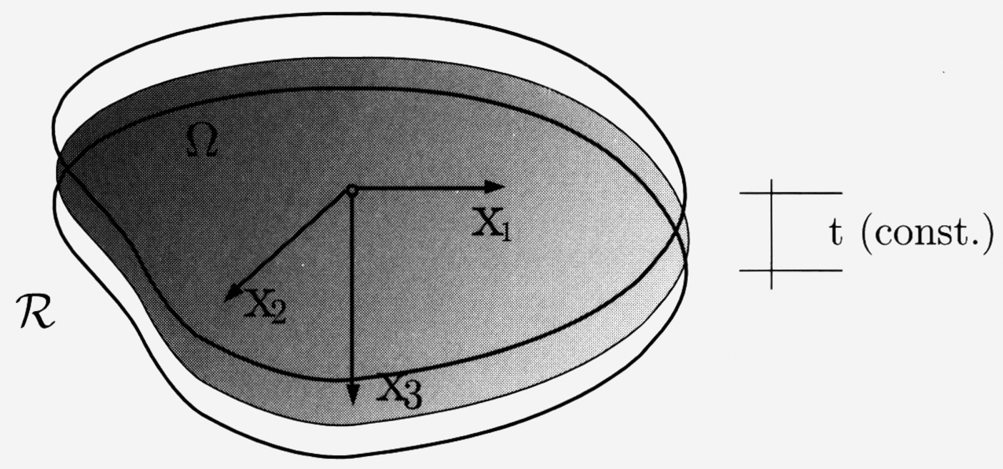

FIG. 1. Cylindrical slab 
where the mapping $\boldsymbol{\varphi}(\mathbf{x})$, known as a deformation, is smooth enough, injective and orientation-preserving, in the sense that $\operatorname{det} \mathbf{D} \varphi(x)>0$ for all $\mathbf{x} \in \overline{\mathcal{R}}(\mathbf{D} \varphi=: \mathbf{F}: \overline{\mathcal{R}} \rightarrow$ $\mathrm{Lin}^{+}$denotes the deformation gradient ${ }^{1}$ ).

We shall use the Piola transform $\mathbf{T}_{\mathbb{R}}: \overline{\mathcal{R}} \rightarrow$ Lin of a tensor field $\mathbf{T}: \varphi(\overline{\mathcal{R}}) \rightarrow$ Sym by letting

$$
\mathbf{T}_{\mathbb{R}}(\mathbf{x}):=\mathbf{T}(\boldsymbol{\varphi}(\mathbf{x})) \operatorname{Cof}(\mathbf{D} \varphi(\mathbf{x})),
$$

where $\mathbf{T}_{\mathbb{R}}$ and $\mathbf{T}$ are the so-called Piola-Kirchhoff and Cauchy stress tensors and Cof $\mathbf{F}=$ $(\operatorname{det} \mathbf{F}) \mathbf{F}^{-\mathrm{T}}$.

For the stored energy function $\widehat{\mathcal{W}}: \overline{\mathcal{R}} \times \operatorname{Lin}^{+} \rightarrow \mathbb{R}$ of a hyperelastic, frame-indifferent and isotropic material, the following property holds:

$$
\widehat{\mathcal{W}}(\mathbf{x}, \mathbf{F})=\widetilde{\mathcal{W}}\left(\mathbf{x}, \iota_{\mathbf{F}^{\mathrm{T}} \mathbf{F}}\right)=\widetilde{\mathcal{W}}\left(\mathbf{x}, \iota_{\mathbf{F}} \mathbf{F}^{\mathrm{T}}\right), \quad \text { for all } \mathbf{F} \in \operatorname{Lin}^{+},
$$

where $\mathbf{C}:=\mathbf{F}^{\mathrm{T}} \mathbf{F}$ and $\mathbf{B}:=\mathbf{F F}^{\mathrm{T}}$ are the right and left Cauchy-Green deformation tensors, while $\iota$ represents, equivalently, the triple formed by the three principal invariants of the tensor $\mathbf{C}$ or $\mathbf{B}$ :

$$
\begin{aligned}
& \iota_{1}=\|\mathbf{F}\|^{2}=\lambda_{1}^{2}+\lambda_{2}^{2}+\lambda_{3}^{2}, \\
& \iota_{2}=\|\operatorname{Cof} \mathbf{F}\|^{2}=\lambda_{1}^{2} \lambda_{2}^{2}+\lambda_{1}^{2} \lambda_{3}^{2}+\lambda_{2}^{2} \lambda_{3}^{2}, \\
& \iota_{3}=(\operatorname{det} \mathbf{F})^{2}=\lambda_{1}^{2} \lambda_{2}^{2} \lambda_{3}^{2}=\delta^{2},
\end{aligned}
$$

with $\|\mathbf{A}\|^{2}=\operatorname{tr} \mathbf{A}^{\mathrm{T}} \mathbf{A} . \quad \lambda_{1}, \lambda_{2}$, and $\lambda_{3}$ are the principal stretches. By dropping the dependence on $\mathbf{x} \in \overline{\mathcal{R}}$, in that the material is supposed to be homogeneous, the associated response function to the stored energy function is given by $\left(\widehat{\mathbf{T}}_{\mathbb{R}}(\mathbf{F})=\frac{\partial \overline{\mathcal{W}}}{\partial \mathbf{F}}(\mathbf{F})\right)$

$$
\frac{1}{2} \widehat{\mathbf{T}}_{\mathbb{R}}(\mathbf{F})=\frac{\partial \widetilde{\mathcal{W}}}{\partial \iota_{1}} \mathbf{F}+\frac{\partial \widetilde{\mathcal{W}}}{\partial \iota_{2}}\left(\iota_{1} \mathbf{I}-\mathbf{B}\right) \mathbf{F}+\frac{1}{2} \frac{\partial \widetilde{\mathcal{W}}}{\partial \delta} \delta \mathbf{F}^{-\top},
$$

where $\mathbf{I}$ is the identity tensor.

The discussion will, on the whole, be confined to a material that has the compressible neo-Hookean form for the stored energy function [33]

$$
\widetilde{\mathcal{W}}(\mathbf{F}):=a\|\mathbf{F}\|^{2}+\Gamma(\operatorname{det} \mathbf{F}), \quad \text { for all } \mathbf{F} \in \operatorname{Lin}^{+},
$$

with $a>0$ and $\Gamma:] 0, \infty[\rightarrow \mathbb{R}$ being a convex function that satisfies the additional assumption $\lim _{\delta \rightarrow 0^{+}} \Gamma(\delta) \rightarrow+\infty$. The last requirement reflects the intuitive idea that "an infinite stored energy is needed to annihilate a volume" [34], [35], and [36]. The expression proposed by Ciarlet and Geymonat [37] is assumed for the function $\Gamma(\delta)$ :

$$
\Gamma(\delta):=c \delta^{2}-d \log \delta
$$

with $c>0, d>0$.

Once the form of the function $\widetilde{\mathcal{W}}(\mathbf{F})$ has been assigned, from (2.2) it follows that

$$
\mathbf{T}_{\mathbb{R}}(\mathbf{F})=2 a \mathbf{F}+\Gamma^{\prime}(\delta) \delta \mathbf{F}^{-\mathrm{T}},
$$

where $\Gamma^{\prime}=\frac{d \Gamma}{d \delta}$.

\footnotetext{
${ }^{1} \mathrm{Lin}^{+}$is the set of all the (second-order) tensors with positive determinant and Sym is the set of those symmetrics. $\mathbb{R}$ denotes the set of real numbers.
} 
To maintain a state of plane stress, the components of the deformation gradient $\mathbf{F}$ are found to be $[28]^{2}$

$$
F_{\alpha \beta}=\varphi_{\alpha, \beta}, \quad F_{\alpha 3}=F_{3 \gamma}=0, \quad F_{33}=\varphi_{3,3}=\lambda\left(x_{1}, x_{2}\right) .
$$

The last quantity denotes the principal stretch with the $x_{3}$-axis as the associated principal axis. To compute $\lambda$ we can use two positive invariants defined on the mid-plane domain $\Omega$, i.e.,

$$
h:=\operatorname{tr}\left(B_{\alpha \beta}\right)=\lambda_{1}^{2}+\lambda_{2}^{2}, \quad j:=\operatorname{det}\left(F_{\alpha \beta}\right)=\lambda_{1} \lambda_{2},
$$

which are linked to those of the three-dimensional problem by the following relations:

$$
\iota_{1}=h+\lambda^{2}, \quad \iota_{2}=j^{2}+h \lambda^{2}, \quad \delta=j \lambda,
$$

and successively impose the approximate assumption $T_{\mathbb{R}_{33}}=0$ (dictated by the plane stress condition), obtaining

$$
\lambda=\left(\frac{d}{2\left(a+c j^{2}\right)}\right)^{1 / 2} .
$$

Equations (2.4), (2.5), and (2.6c) can be employed to express the non-vanishing components of the Piola-Kirchhoff stress tensor $\left(T_{\mathbb{R}_{\alpha 3}}=T_{\mathbb{R}_{3, \beta}}=T_{\mathbb{R}_{33}}=0\right)$

$$
T_{\mathbb{R}_{\alpha \beta}}=2 a F_{\alpha \beta}+\left[2 c j \lambda^{2}-\frac{d}{j}\right] \varepsilon_{\alpha \mu} \varepsilon_{\beta \nu} F_{\mu \nu}
$$

where $F_{\alpha \beta}^{-\mathrm{T}}=j^{-1}(\operatorname{Cof} F)_{\alpha \beta}=j^{-1} \varepsilon_{\alpha \mu} \varepsilon_{\beta \nu} F_{\mu \nu}$ (the symbol $\varepsilon_{\alpha \mu}$ indicates the two-dimensional alternator, i.e., $\left.\varepsilon_{11}=\varepsilon_{22}=0, \varepsilon_{12}=-\varepsilon_{21}=1\right)$. By substituting this expression into the equation of equilibrium in the absence of body forces, i.e.,

$$
\operatorname{Div} \mathbf{T}_{\mathbb{R}}=\mathbf{0},
$$

the following equations in terms of spatial coordinates $\varphi_{\alpha}\left(x_{1}, x_{2}\right)$ are derived:

$$
\begin{aligned}
& -2 a \Delta \varphi_{1}=\psi_{.1} \varphi_{2.2}-\psi_{, 2} \varphi_{2.1}, \\
& -2 a \Delta \varphi_{2}=-\psi_{.1} \varphi_{1,2}+\psi_{.2} \varphi_{1,1},
\end{aligned}
$$

where

$$
\begin{gathered}
h=\varphi_{1,1}^{2}+\varphi_{1,2}^{2}+\varphi_{2,1}^{2}+\varphi_{2,2}^{2}, \quad j=\varphi_{1,1} \varphi_{2,2}-\varphi_{1,2} \varphi_{2,1}, \\
\lambda^{2}=\frac{d}{2\left(a+c j^{2}\right)}, \quad \psi=2 c j \lambda^{2}-\frac{d}{j},
\end{gathered}
$$

and $\Delta$ denotes the two-dimensional Laplace operator. The elastostatic finite plane stress problem for a compressible neo-Hookean material is governed by the above nonlinear system of coupled partial differential equations.

\footnotetext{
${ }^{2}$ Under the plane stress condition, the functions, initially defined on the region $\mathcal{R}$, must be considered as their restriction to the plane $\Omega$.
} 
3. Mathematical formulation of the wedge problem. With a view to formulating wedge analyses, it is convenient to express field equations and boundary conditions by means of the complex spatial coordinate

$$
\varphi(r, \theta)=\varphi_{1}(r, \theta)+i \varphi_{2}(r, \theta), \quad r>0,-\pi \leq \theta \leq \pi,
$$

which depends on the material polar coordinates $(r, \theta)$. The equilibrium equations $(2.9 \mathrm{a}, \mathrm{b})$ thus transform into

$$
-2 a \Delta \varphi=\frac{i}{r}\left(\psi_{. \theta} \boldsymbol{\varphi}_{. r}-\psi_{. r} \boldsymbol{\varphi}_{. \theta}\right),
$$

where $\Delta \varphi=\varphi_{. r r}+\frac{1}{r} \varphi_{. r}+\frac{1}{r^{2}} \varphi_{. \theta \theta}$ and the invariants $h$ and $j$ become

$$
h=\left|\boldsymbol{\varphi}_{. r}\right|^{2}+\frac{1}{r^{2}}\left|\boldsymbol{\varphi}_{. \theta}\right|^{2} \quad \text { and } \quad j=\frac{1}{r} \operatorname{Im}\left\{\overline{\boldsymbol{\varphi}}_{. r} \boldsymbol{\varphi}_{. \theta}\right\} .
$$

Here $|\mathbf{a}|^{2}=\mathbf{a} \cdot \overline{\mathbf{a}}$ denotes the Hermitian product, the bar denotes the complex conjugate and Im denotes the imaginary part. Whereas, the constitutive equations lead to the following relations:

$$
\begin{aligned}
& T_{\mathbb{R}_{11}}+i T_{\mathbb{R}_{21}}=\varphi_{. r}(2 a \cos \theta-i \psi \sin \theta)-\frac{1}{r} \varphi_{. \theta}(2 a \sin \theta+i \psi \cos \theta), \\
& T_{\mathbb{R}_{12}}+i T_{\mathbb{R}_{22}}=\varphi_{. r}(2 a \sin \theta+i \psi \cos \theta)+\frac{1}{r} \varphi_{. \theta}(2 a \cos \theta-i \psi \sin \theta),
\end{aligned}
$$

from which the (non-vanishing) polar components of the Piola-Kirchhoff stress tensor can be deduced:

$$
\begin{aligned}
& T_{\mathbb{R}_{r},}+i T_{\mathbb{R}_{\theta r}}=\left(2 a \varphi_{. r}-i \psi \frac{1}{r} \varphi_{. \theta}\right) e^{-i \theta}, \\
& T_{\mathbb{E}_{, \theta}}+i T_{\mathbb{R}_{\theta \theta}}=\left(2 a \frac{1}{r} \varphi_{. \theta}+i \psi \varphi_{. r}\right) e^{-i \theta} .
\end{aligned}
$$

We are now in position to define a wedge problem. The infinitely extended slab illustrated by Fig. 1 is considered as the body in its undeformed configuration. In the case of a standard wedge, the mid-plane domain $\Omega$ of this slab is described by

$$
\Omega=\{(r, \theta) \mid 0<r<\infty, \bar{\theta} \leq \theta \leq \pi \text {, and } 0 \leq \bar{\theta}<\pi\},
$$

where $(r, \theta)$ are the material polar coordinates of any point in the mid-plane of the wedge with respect to the origin 0 at the wedge vertex (Fig. 2). The wedge angle is designated by $\phi=\pi-\bar{\theta}$. By increasing this angle, the possibility of the wedge faces coalescing until they form an interface is admitted. This situation is depicted by the reentrant wedge model shown in Fig. 3, where

$$
\Omega=\{(r, \theta) \mid 0<r<\infty, 0 \leq \theta \leq \pi \text { together with }-\bar{\theta} \leq-\theta \leq 0 \text { and }-\pi<-\bar{\theta} \leq 0\} .
$$

Commonly, the wedge problem is formulated locally. This can be stated as follows. Find a suitably regular deformation $\varphi(r, \theta)$, with $(r, \theta) \in \Omega$, that satisfies the field equations (3.1) and that is consistent with the following two kinds of boundary conditions.

- At least in proximity of the vertex, the displacement ${ }^{3}$ of points lying along the radial clamped edge $(\theta= \pm \bar{\theta})$ vanishes, viz.,

$$
\varphi(r, \pm \bar{\theta})=\operatorname{id}(r, \pm \bar{\theta})
$$

\footnotetext{
${ }^{3}$ The displacement is the vector field $\mathbf{u}: \bar{\Omega} \rightarrow \mathbb{R}^{2}$ defined by the relation $\mathbf{u}=\boldsymbol{\varphi}-\mathbf{i d}$, where id denotes the (restriction to $\bar{\Omega}$ of the) identity map from $\mathbb{R}^{2}$ onto $\mathbb{R}^{2}$.
} 


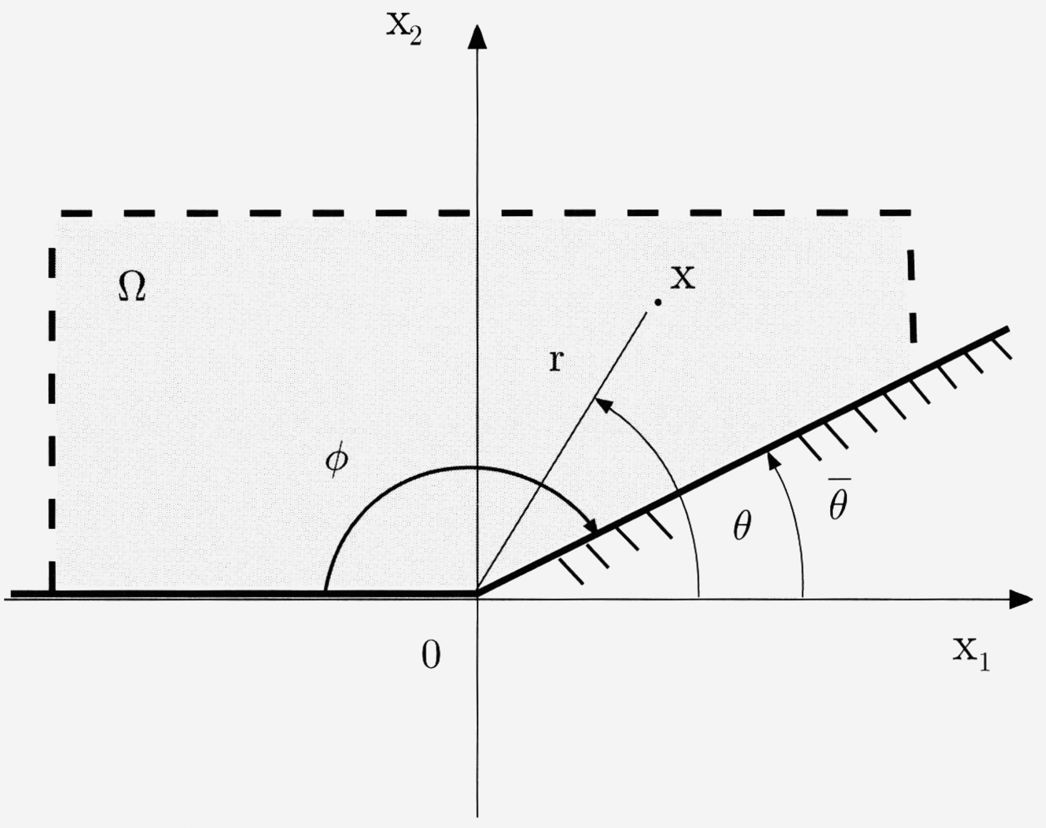

FIG. 2. The standard wedge model

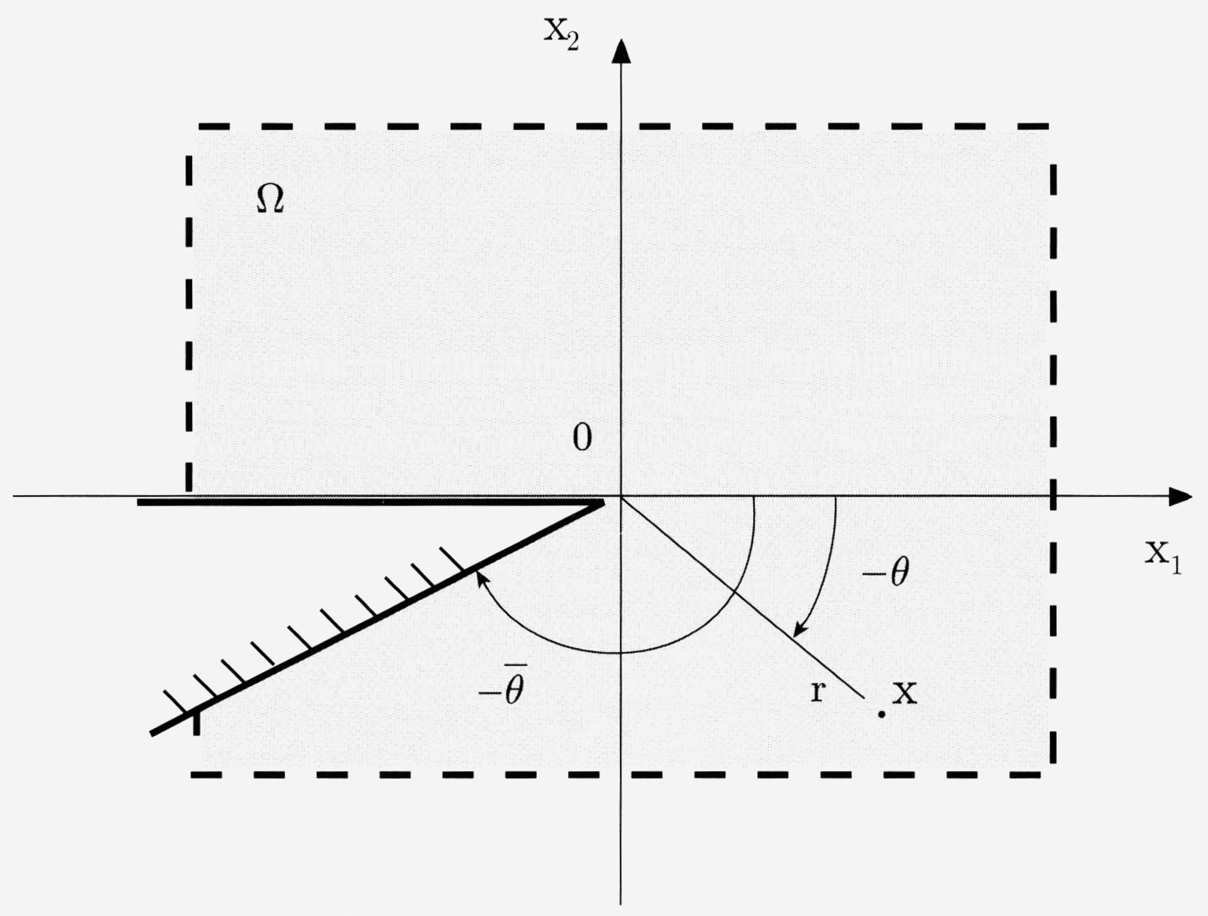

FIG. 3. The reentrant wedge model 
- Through (2.8), such a deformation generates a Piola-Kirchhoff stress field satisfying the condition that the free radial edge $(\theta=\pi)$ be (at least in proximity of the vertex) traction-free.

It is important to note that the Piola-Kirchhoff stress tensor possesses the following fundamental property. Let $\mathcal{C}$ be a regular arc in $\Omega$ and $\mathcal{C}^{*}$ its image in the deformed configuration, and let $\mathbf{n}, \mathbf{n}^{*}$ be the orienting unit normal vectors of $\mathcal{C}$ and $\mathcal{C}^{*}$, respectively. The Piola transform implies that

$$
\mathbf{T n}^{*}=\mathbf{0} \text { on } \mathcal{C}^{*} \quad \text { if and only if } \quad \mathbf{T}_{\mathbb{B}} \mathbf{n}=\mathbf{0} \text { on } \mathcal{C} .
$$

It is hence possible to impose the traction-free boundary condition without a priori knowledge of the deformation.

Equation (3.4b) can therefore be used to impose this latter condition:

$$
\left.\left(T_{\mathbb{R}_{, \theta}}+i T_{\mathbb{R}_{\theta \theta}}\right)\right|_{\theta=\pi}=0
$$

or, equivalently in terms of the deformation,

$$
\left.\left(2 a \frac{1}{r} \varphi_{. \theta}+i \psi \varphi_{. r}\right)\right|_{\theta=\pi}=0 .
$$

At the infinite edge, the deformation must be compatible with kinematic loading conditions that, in the local formulation, are left unspecified. ${ }^{4}$ We only demand that these far-field loading conditions can induce, for certain vertex angles, singular solutions at the wedge-apex.

Note that, without corresponding requirements on the deformation field at infinity the above formulation is not a complete statement. However, the significance of the fields admitted by the local formulation lies in their characterisation of the singular elastostatic field behaviour in the corner region, namely, as $r \rightarrow 0$.

4. Asymptotic deformation field. In this section an asymptotic procedure will be developed in order to solve the local problem stated in Sec. 3.

Supposing that the global wedge problem admits a solution, to investigate the singular behaviour at the apex, we assume that such a solution has the following asymptotic representation form:

$$
\varphi_{\alpha}(r, \theta)=r^{m_{\circ}} v_{\alpha}(\theta)+o\left(r^{m_{n}}\right)^{5} \quad \text { as } r \rightarrow 0 \quad(\text { no sum on } \alpha),
$$

where $m_{\alpha}$ denote two unknown real-valued constants ${ }^{6}$ obeying the inequality

$$
0<m_{a}<1 .
$$

The exponents $m_{\alpha}$ are positive; they must in fact not be less than zero or the displacement would become infinite as $r \rightarrow 0$. While the assumption $m_{\alpha}<1$ ensures that the deformation gradient does not remain bounded as $r \rightarrow 0$. The functions $v_{\alpha}(\theta)$ are twice continuously differentiable real-valued unknown functions that fail to vanish identically on $[ \pm \bar{\theta}, \pi]$.

\footnotetext{
${ }^{4}$ Consequently, in the solution a number of undetermined constants will appear.

${ }^{5} O(\cdot)$ and $O(\cdot)$ are the Landau order symbols.

${ }^{6}$ Given the kind of boundary condition (3.5), the exponents $m_{\alpha}$ are supposed to be different in the formulation. This is contrary to nonlinear ciack analyses or to notch problems (see, for example, [23], [38], and [21]), where, without loss of generality, it is possible to assume directly that the two constants coincide (as observed by Stephenson [39]).
} 
The asymptotic analysis can hence be performed by determining the smallest exponents $\left.m_{\alpha} \in\right] 0,1\left[\right.$ and the angular functions $v_{\alpha}(\theta)$ consistent with the equilibrium equations (3.1) and the boundary conditions (3.5) and (3.6). Demanding the validity of the asymptotic equalities resulting from differentiations of (4.1) with respect to the polar coordinates $r$ and $\theta$ and inserting these into (3.2b) and (2.7), we obtain

$$
\begin{gathered}
j(r, \theta)=\frac{1}{r}\left\{\varphi_{1 . r} \varphi_{2 . \theta}-\theta_{1 . \theta} \varphi_{2, r}\right\}=r^{\left(m_{1}+m_{2}-2\right)} M(\theta)+o\left(r^{\left(m_{1}+m_{2}-2\right)}\right), \\
\lambda^{2}(r, \theta)=\frac{d}{2\left(a+c r^{2\left(m_{1}+m_{2}-2\right)} M^{2}(\theta)\right)}+o\left(r^{2\left(2-m_{1}-m_{2}\right)}\right),
\end{gathered}
$$

where $M(\theta)=m_{1} v_{1}(\theta) \dot{v}_{2}(\theta)-m_{2} \dot{v}_{1}(\theta) v_{2}(\theta)$ (the dot denotes differentiation with respect to $\theta$ ). Operating in the same way, the field equations (3.1) transform into

$$
\begin{gathered}
-2 a\left[m_{1}^{2} v_{1}(\theta)+\ddot{v}_{1}(\theta)\right] r^{\left(m_{1}-2\right)}+o\left(r^{\left(m_{1}-2\right)}\right) \\
=\left[\dot{v}_{2}(\theta) r \psi_{. r}-m_{2} v_{2}(\theta) \psi_{. \theta}\right] r^{\left(m_{2}-2\right)}, \\
-2 a\left[m_{2}^{2} v_{2}(\theta)+\ddot{v}_{2}(\theta)\right] r^{\left(m_{2}-2\right)}+o\left(r^{\left(m_{2}-2\right)}\right) \\
=\left[m_{1} v_{1}(\theta) \psi_{, \theta}-\dot{v}_{1}(\theta) r \psi_{. r}\right] r^{\left(m_{1}-2\right)},
\end{gathered}
$$

where

$$
\begin{gathered}
r \psi_{, r}=\frac{c d\left(m_{1}+m_{2}-2\right) r^{\left(m_{1}+m_{2}-2\right)} M(\theta)}{\left(a+c r^{2\left(m_{1}+m_{2}-2\right)} M^{2}(\theta)\right)}-\frac{2 c^{2} d\left(m_{1}+m_{2}-2\right) r^{3\left(m_{1}+m_{2}-2\right)} M^{3}(\theta)}{\left(a+c r^{2\left(m_{1}+m_{2}-2\right)} M^{2}(\theta)\right)^{2}} \\
+\frac{d\left(m_{1}+m_{2}-2\right)}{r^{\left(m_{1}+m_{2}-2\right)} M(\theta)}+o\left(r^{2-m_{1}-m_{2}}\right), \\
\psi_{, \theta}=\frac{c d r^{\left(m_{1}+m_{2}-2\right)} \dot{M}(\theta)}{\left(a+c r^{2\left(m_{1}+m_{2}-2\right)} M^{2}(\theta)\right)}-\frac{2 c^{2} d r^{3\left(m_{1}+m_{2}-2\right)} M^{2}(\theta) \dot{M}(\theta)}{\left(a+c r^{2\left(m_{1}+m_{2}-2\right)} M^{2}(\theta)\right)^{2}} \\
\quad+\frac{d \dot{M}(\theta)}{r^{\left(m_{1}+m_{2}-2\right)} M^{2}(\theta)}+o\left(r^{\left.2-m_{1}-m_{2}\right)} .\right.
\end{gathered}
$$

Dividing Eq. (4.3a) by $r^{\left(m_{1}-2\right)}$ and Eq. (4.3b) by $r^{\left(m_{2}-2\right)}$ and proceeding to the limit as $r \rightarrow 0$, one readily arrives at

$$
m_{\alpha}^{2} v_{\alpha}(\theta)+\ddot{v}_{\alpha}(\theta)=0 \quad(\text { no sum on } \alpha) .
$$

In fact, $r \psi_{. r}$ and $\psi_{. \theta}$ behave like $O\left(r^{2-m_{1}-m_{2}}\right)$ as $r \rightarrow 0$ and $\left.m_{\alpha} \in\right] 0,1[$.

Analogously, for the boundary condition (3.6) we have

$$
\begin{aligned}
& 2 a r^{\left(m_{1}-1\right)} \dot{v}_{1}(\pi)-m_{2} \psi(r, \pi) r^{\left(m_{2}-1\right)} v_{2}(\pi)+o\left(r^{\left(m_{1}-1\right)}\right)=0, \\
& 2 a r^{\left(m_{2}-1\right)} \dot{v}_{2}(\pi)+m_{1} \psi(r, \pi) r^{\left(m_{1}-1\right)} v_{1}(\pi)+o\left(r^{\left(m_{2}-1\right)}\right)=0,
\end{aligned}
$$

where

$$
\psi(r, \theta)=\frac{d}{r^{\left(m_{1}+m_{2}-2\right)}}\left[\frac{c M(\theta)}{a r^{-2\left(m_{1}+m_{2}-2\right)}+c M^{2}(\theta)}-\frac{1}{M(\theta)}\right]+o\left(r^{2-m_{1}-m_{2}}\right) .
$$


Dividing Eq. (4.5a) by $r^{\left(m_{1}-1\right)}$ and Eq. (4.5b) by $r^{\left(m_{2}-1\right)}$ and proceeding to the limit as $r \rightarrow 0$, we obtain

$$
\dot{v}_{\alpha}(\pi)=0,
$$

because $\psi$ behaves like $O\left(r^{2-m_{1}-m_{2}}\right)$ as $r \rightarrow 0$ and $\left.m_{\alpha} \in\right] 0,1[$.

With the use of (4.1), the boundary condition (3.5) can be expressed as

$$
\begin{aligned}
& r^{m_{1}} v_{1}(\bar{\theta})+o\left(r^{m_{1}}\right)=r \cos \bar{\theta}, \\
& r^{m_{2}} v_{2}(\bar{\theta})+o\left(r^{m_{2}}\right)=r \sin \bar{\theta} .
\end{aligned}
$$

Dividing Eq. (4.7a) by $r^{m_{1}}$ and Eq. (4.7b) by $r^{m_{2}}$ and proceeding to the limit as $r \rightarrow 0$, we obtain

$$
v_{\alpha}(\bar{\theta})=0,
$$

since $\left.m_{\alpha} \in\right] 0,1[$.

The eigenvalue problem, described by (4.4), (4.6), and (4.8), constitutes two uncoupled boundary-value problems for the determination of the two scalar components of the asymptotic deformation field. The solutions of (4.4) assume the form

$$
v_{\alpha}(\theta)=A_{\alpha} \sin m_{\alpha} \theta+C_{\alpha} \cos m_{\alpha} \theta \quad \text { (no sum on } \alpha \text { ), }
$$

and the boundary conditions can then be used to determine the eigenvalues $m_{\alpha}$. In fact, by introducing (4.9) into (4.6) and (4.8) we obtain

$$
\begin{gathered}
A_{\alpha} m_{\alpha} \cos m_{\alpha} \pi-C_{\alpha} m_{\alpha} \sin m_{\alpha} \pi=0, \\
A_{\alpha} \sin m_{\alpha} \bar{\theta}+C_{\alpha} \cos m_{\alpha} \bar{\theta}=0 \quad(\text { no sum on } \alpha) .
\end{gathered}
$$

Since $m_{\alpha} \neq 0$ and $A_{\alpha}=C_{\alpha}=0$ are ruled out by the condition $v_{\alpha}(\theta) \neq 0$ on $[ \pm \bar{\theta}, \pi]$, the homogeneous system $(4.10 \mathrm{a}, \mathrm{b})$ admits solutions different from the trivial solution if and only if

$$
\cos m_{\alpha}(\pi-\bar{\theta})=0 .
$$

It follows that the smallest positive eigenvalue for both the boundary-value problems is $m=\frac{\pi}{2(\pi-\bar{\theta})}$ and the associated eigenfunctions are

$$
v_{\alpha}(\theta)=\frac{A_{\alpha}}{\cos m \bar{\theta}} \sin m(\theta-\bar{\theta}),
$$

where $A_{\alpha}$ denote two arbitrary real constants. However, the inequality $m<1$ implies $\bar{\theta}<\frac{\pi}{2}$. Namely, the first asymptotic analysis provides unbounded deformation gradients for wedges with a vertex angle $\phi$ larger than $\frac{\pi}{2}$.

Therefore, the first asymptotic solution is

$$
\begin{aligned}
\varphi_{\alpha}(r, \theta)= & r^{\frac{\pi}{2(\pi-\bar{\theta})}} \frac{A_{\alpha}}{\cos \left(\frac{\pi \bar{\theta}}{2(\pi-\bar{\theta})}\right)} \sin \frac{\pi(\theta-\bar{\theta})}{2(\pi-\bar{\theta})}+o\left(r^{\left.\frac{\pi}{2(\pi-\bar{\theta})}\right)}\right. \\
& \text { as } r \rightarrow 0,0 \leq \bar{\theta}<\frac{\pi}{2} \text { and } \bar{\theta} \leq \theta \leq \pi \text { (standard wedge) or } \\
& -\pi \leq-\bar{\theta} \leq 0 \text { and } 0 \leq \theta \leq \pi \text { together with }-\bar{\theta} \leq-\theta \leq 0 \text { (reentrant wedge). }
\end{aligned}
$$


Nevertheless, such a solution implies $M(\theta) \equiv 0$, providing the following weak estimate:

$$
j(r, \theta) \cong o\left(r^{\frac{2 \bar{\theta}-\pi}{\pi-\bar{\theta}}}\right),
$$

which is inadequate. Since $\delta=j \lambda \cong o\left(r^{(2 \bar{\theta}-\pi) /(\pi-\bar{\theta})}\right)$, the lowest-order approximation to the local deformation in the vicinity of the vertex of the wedge does not constitute an invertible mapping. Consequently, we should refine (4.1) by seeking at least a two-term approximation:

$$
\varphi_{\alpha}(r, \theta)=r^{m} v_{\alpha}(\theta)+r^{m_{\alpha}^{\prime}} w_{\alpha}(\theta)+o\left(r^{m_{\alpha}^{\prime}}\right) \quad \text { as } r \rightarrow 0 \quad(\text { no sum on } \alpha),
$$

where $m_{\alpha}^{\prime}>m$ and $w_{\alpha}(\theta)$ are as yet undetermined, whereas the functions $v_{\alpha}(\theta)$ and the exponent $m$ are known from the above computations. Like the first asymptotic analysis, $w_{\alpha}(\theta)$ are twice continuously differentiable real-valued functions that fail to vanish identically on $[ \pm \bar{\theta}, \pi]$.

By using (4.13), the foregoing asymptotic procedure can be parallelly repeated. Eq. $(4.2 \mathrm{a})$ is then replaced by

$$
j(r, \theta)=Q_{1}(\theta) r^{\left(m+m_{1}^{\prime}-2\right)}+Q_{2}(\theta) r^{\left(m+m_{2}^{\prime}-2\right)}+M^{\prime}(\theta) r^{\left(m_{1}^{\prime}+m_{2}^{\prime}-2\right)}+o\left(r^{\left(m_{1}^{\prime}+m_{2}^{\prime}-2\right)}\right),
$$

where

$$
\begin{aligned}
& Q_{1}(\theta)=m_{1}^{\prime} \dot{v}_{2}(\theta) w_{1}(\theta)-m v_{2}(\theta) \dot{w}_{1}(\theta), \\
& Q_{2}(\theta)=m v_{1}(\theta) \dot{w}_{2}(\theta)-m_{2}^{\prime} \dot{v}_{1}(\theta) w_{2}(\theta), \\
& M^{\prime}(\theta)=m_{1}^{\prime} w_{1}(\theta) \dot{w}_{2}(\theta)-m_{2}^{\prime} \dot{w}_{1}(\theta) w_{2}(\theta),
\end{aligned}
$$

and functions $\lambda^{2}(r, \theta)$ and $\psi(r, \theta)$ can be re-evaluated. Bearing in mind that functions $v_{\alpha}(\theta)$ already satisfy the asymptotic equilibrium conditions, (3.1) can be expressed as

$$
\begin{gathered}
-2 a\left[m_{1}^{\prime 2} w_{1}(\theta)+\ddot{w}_{1}(\theta)\right] r^{\left(m_{1}^{\prime}-2\right)}+o\left(r^{\left(m_{1}^{\prime}-2\right)}\right) \\
=\left[r \psi_{. r} \dot{w}_{2}(\theta)-m_{2}^{\prime} \psi_{, \theta} w_{2}(\theta)\right] r^{\left(m_{2}^{\prime}-2\right)}, \\
-2 a\left[m_{2}^{\prime 2} w_{2}(\theta)+\ddot{w}_{2}(\theta)\right] r^{\left(m_{2}^{\prime}-2\right)}+o\left(r^{\left(m_{2}^{\prime}-2\right)}\right) \\
=\left[m_{1}^{\prime} \psi_{. \theta} w_{1}(\theta)-r \psi_{. r} \dot{w}_{1}(\theta)\right] r^{\left(m_{1}^{\prime}-2\right)} .
\end{gathered}
$$

We now divide Eq. (4.15a) by $r^{\left(m_{1}^{\prime}-2\right)}$ and Eq. $(4.15 \mathrm{~b})$ by $r^{\left(m_{2}^{\prime}-2\right)}$ and proceed to the limit as $r \rightarrow 0$. Supposing temporarily that $m_{\alpha}^{\prime}<2-m$, the system $(4.15 \mathrm{a}, \mathrm{b})$ is asymptotically balanced if

$$
m_{\alpha}^{\prime 2} w_{\alpha}(\theta)+\ddot{w}_{\alpha}(\theta)=0 \quad(\text { no sum on } \alpha) .
$$

In fact as $r \rightarrow 0, r \psi_{, r}$ and $\psi_{, \theta}$ behave like $O\left(r^{\left(2-m-m_{1}^{\prime}\right)}\right)$ if $m_{2}^{\prime} \geq m_{1}^{\prime}$ and like $O\left(r^{\left(2-m-m_{2}^{\prime}\right)}\right)$ if $m_{1}^{\prime} \geq m_{2}^{\prime}$ and in both situations, however, the nonlinear terms, which appear on the right-hand sides of (3.1), once again fail to enter the asymptotic analysis explicitly.

Likewise, substituting (4.13) in the boundary condition (3.6), this is asymptotically balanced if

$$
\dot{w}_{\alpha}(\pi)=0 .
$$


To satisfy the boundary condition (3.5) regarding the clamped edge, i.e.,

$$
\begin{aligned}
& r^{m} v_{1}(\bar{\theta})+r^{m_{1}^{\prime}} w_{1}(\bar{\theta})+o\left(r^{m_{1}^{\prime}}\right)=r \cos \bar{\theta}, \\
& r^{m} v_{2}(\bar{\theta})+r^{m_{2}^{\prime}} w_{2}(\bar{\theta})+o\left(r^{m_{2}^{\prime}}\right)=r \sin \bar{\theta},
\end{aligned}
$$

two possibilities exist. Considering Eq. (4.8), we divide Eq. (4.18a) by $r^{m_{1}^{\prime}}$ and Eq. (4.18b) by $r^{m_{2}^{\prime}}$ and proceed to the limit as $r \rightarrow 0$. Thus, if $m_{\alpha}^{\prime}<1$, we obtain

$$
w_{\alpha}(\bar{\theta})=0 \text {. }
$$

On the other hand, setting $m_{\alpha}^{\prime}=1$, relations $(4.18 \mathrm{a}, \mathrm{b})$ are asymptotically satisfied when

$$
w_{1}(\bar{\theta})=\cos \bar{\theta}, \quad w_{2}(\bar{\theta})=\sin \bar{\theta} .
$$

Note that uncoupled eigenvalue problems are again derived. The two boundary-value problems, described by (4.16), (4.17), and (4.19), admit the solutions

$$
w_{\alpha}^{\prime}(\theta)=\frac{B_{\alpha}}{\cos m^{\prime} \bar{\theta}} \sin m^{\prime}(\theta+\bar{\theta}), \quad m^{\prime}=\frac{3 \pi}{2(\pi+\bar{\theta})}
$$

which, given the limitations $m_{\alpha}^{\prime}<1$, hold only in the interval $-\pi \leq-\bar{\theta}<-\frac{\pi}{2}$. In (4.21a), $B_{\alpha}$ denote two arbitrary real constants. The two boundary-value problems, described by (4.16), (4.17), and (4.20a,b), yield

$$
w_{1}^{\prime \prime}(\theta)=\cos \theta, \quad w_{2}^{\prime \prime}(\theta)=\tan \bar{\theta} \cos \theta
$$

The requirement $m<m_{\alpha}^{\prime}=1$ implies, for the angle $\bar{\theta}$ in $(4.22 \mathrm{~b})$, the same limitations of the solution (4.12). Both solutions (4.21a) and (4.22a,b) satisfy the inequalities $m_{\alpha}^{\prime}<$ $2-m$, which lead to Eqs. (4.16) and (4.17).

We are now in position to write, for the intervals $0 \leq \bar{\theta}<\frac{\pi}{2}$ and $-\frac{\pi}{2}<-\bar{\theta} \leq 0$, a two-term asymptotic solution

$$
\begin{aligned}
& \varphi_{\alpha}(r, \theta)=r^{m} v_{a}(\theta)+r w_{\alpha}^{\prime \prime}(\theta)+o(r), \\
& \quad \text { as } r \rightarrow 0, \quad \bar{\theta} \leq \theta \leq \pi \text { (standard wedge) or } \\
& \quad 0 \leq \theta \leq \pi \text { together with }-\bar{\theta} \leq-\theta \leq 0 \text { (reentrant wedge) },
\end{aligned}
$$

and, for the interval $-\pi \leq-\bar{\theta}<-\frac{\pi}{2}$, a three-term asymptotic solution

$$
\begin{aligned}
\varphi_{\alpha}(r, \theta)=r^{m} v_{\alpha}(\theta)+r^{m^{\prime}} w_{\alpha}^{\prime}(\theta) & +r w_{\alpha}^{\prime \prime}(\theta)+o(r), \\
& \text { as } r \rightarrow 0, \quad 0 \leq \theta \leq \pi \text { together with }-\bar{\theta} \leq-\theta \leq 0 .
\end{aligned}
$$

To perform a three-term asymptotic analysis, the inequality $m^{\prime}<m^{\prime \prime}=1$, in addition to the foregoing restrictions, must be imposed. Such a further condition is however satisfied when the angle $(-\bar{\theta})$ belongs to the interval $-\pi \leq-\bar{\theta}<-\frac{\pi}{2}$.

Solution (4.23) implies

$$
\begin{gathered}
M^{\prime}(\theta) \equiv 0, \\
j(r, \theta)=\frac{m}{\cos m \bar{\theta}}\left(A_{2}-A_{1} \tan \bar{\theta}\right) \cos [(m-1) \theta-m \bar{\theta}] r^{(m-1)}+o\left(r^{(m-1)}\right),
\end{gathered}
$$


$\lambda(r, \theta)=\left(\frac{d}{2\left(a+c \frac{m^{2}}{\cos ^{2} m \bar{\theta}}\left(A_{2}-A_{1} \tan \bar{\theta}\right)^{2} \cos ^{2}[(m-1) \theta-m \bar{\theta}] r^{2(m-1)}\right)}\right)^{1 / 2}+o\left(r^{(1-m)}\right)$.

From the last two equations we can evaluate the determinant of the deformation gradient at the wedge-apex, viz.,

$$
\delta=\left(\frac{d}{2 c}\right)^{1 / 2}>0
$$

showing how the deformation (4.23) now constitutes an invertible and orientation-preserving mapping.

Solution (4.24) provides

$$
\begin{aligned}
j(r, \theta)= & \frac{m m^{\prime}}{\cos m \bar{\theta} \cos m^{\prime} \bar{\theta}}\left(A_{2} B_{1}-A_{1} B_{2}\right) \sin \left[\left(m^{\prime}-m\right)(\theta+\bar{\theta})\right] r^{\left(m+m^{\prime}-2\right)} \\
& +\frac{m}{\cos m \bar{\theta}}\left(A_{2}+A_{1} \tan \bar{\theta}\right) \cos [(m-1) \theta+m \bar{\theta}] r^{(m-1)} \\
& +\frac{m^{\prime}}{\cos m^{\prime} \bar{\theta}}\left(B_{2}+B_{1} \tan \bar{\theta}\right) \cos \left[\left(m^{\prime}-1\right) \theta+m^{\prime} \bar{\theta}\right] r^{\left(m^{\prime}-1\right)}+o\left(r^{\left(m^{\prime}-1\right)}\right)
\end{aligned}
$$

and the same expression (4.26) for the determinant of the deformation gradient at the apex.

In conclusion, when the wedge angle $\phi$ varies from $\frac{1}{2} \pi$ (quarter-plane) to $2 \pi$ (fullplane with a straight crack), solutions (4.23) and $(4.24)^{7}$ provide the deformation fields in proximity of the wedge-apex. From these solutions, unbounded deformation gradients can be derived. In fact, the exponent of the dominant term in (4.23) and (4.24), as the wedge angle $\phi$ varies from $\frac{1}{2} \pi$ to $2 \pi$, correspondingly assumes the numerical values (less than one) belonging to the interval $\left.] 1, \frac{1}{4}\right]$. It is also evident how the order of singularity of the components of the deformation gradient (cf. Eq. 5.3) increases as the wedge angle increases. Thus, the more severe situation occurs for the case of the full-plane with a straight crack. In the solution (4.24), two singular eigenvalues may coexist.

The foregoing asymptotic procedures can be repeated in order to add other higherorder terms to the solutions. Nevertheless, these terms (with eigenvalues larger than $m^{\prime \prime}=1$ ) are inessential to describe the singular behaviour at the wedge apex.

The asymptotic deformation fields obtained are employed, in the next section, to compute the singular stress fields.

5. Asymptotic Piola-Kirchhoff and Cauchy stress fields. Taking into account that the function $\psi(r, \theta)$ behaves like an infinitesimal quantity as $r \rightarrow 0$, asymptotic results for polar components of the Piola-Kirchhoff stress tensor are readily obtainable

\footnotetext{
${ }^{7}$ These solutions, however, deteriorate as $\bar{\theta}$ approaches $\pm \frac{\pi}{2}$.
} 
from (4.24) and (3.4a,b). Thus,

$$
\begin{aligned}
& \begin{aligned}
\frac{T_{\mathbb{R}_{r r}}}{2 a}= & m r^{(m-1)}\left[v_{1}(\theta) \cos \theta+v_{2}(\theta) \sin \theta\right] \\
& \quad+m^{\prime} r^{\left(m^{\prime}-1\right)}\left[w_{1}^{\prime}(\theta) \cos \theta+w_{2}^{\prime}(\theta) \sin \theta\right]+\left[w_{1}^{\prime \prime}(\theta) \cos \theta+w_{2}^{\prime \prime}(\theta) \sin \theta\right]+o(1), \\
\frac{T_{\mathbb{R}_{\theta r}}=}{2 a}= & r^{(m-1)}\left[v_{2}(\theta) \cos \theta-v_{1}(\theta) \sin \theta\right] \\
& \quad+m^{\prime} r^{\left(m^{\prime}-1\right)}\left[w_{2}^{\prime}(\theta) \cos \theta-w_{1}^{\prime}(\theta) \sin \theta\right]+\left[w_{2}^{\prime \prime}(\theta) \cos \theta-w_{1}^{\prime \prime}(\theta) \sin \theta\right]+o(1), \\
\frac{T_{\mathbb{R}_{r} \theta}}{2 a}= & r^{(m-1)}\left[\dot{v}_{1}(\theta) \cos \theta+\dot{v}_{2}(\theta) \sin \theta\right]
\end{aligned} \\
& \quad+r^{\left(m^{\prime}-1\right)}\left[\dot{w}_{1}^{\prime}(\theta) \cos \theta+\dot{w}_{2}^{\prime}(\theta) \sin \theta\right]+\left[\dot{w}_{1}^{\prime \prime}(\theta) \cos \theta+\dot{w}_{2}^{\prime \prime}(\theta) \sin \theta\right]+o(1), \\
& \frac{T_{\mathbb{R}_{\theta \theta}}=}{2 a}=r^{(m-1)}\left[\dot{v}_{2}(\theta) \cos \theta-\dot{v}_{1}(\theta) \sin \theta\right] \\
& \quad+r^{\left(m^{\prime}-1\right)}\left[\dot{w}_{2}^{\prime}(\theta) \cos \theta-\dot{w}_{1}^{\prime}(\theta) \sin \theta\right]+\left[\dot{w}_{2}^{\prime \prime}(\theta) \cos \theta-\dot{w}_{1}^{\prime \prime}(\theta) \sin \theta\right]+o(1) .
\end{aligned}
$$

In the same way, Eqs. $(3.3 \mathrm{a}, \mathrm{b})$ provide the cartesian components

$$
\begin{aligned}
& \frac{T_{\mathbb{R}_{11}}}{2 a}=r^{(m-1)}\left[m v_{1}(\theta) \cos \theta-\dot{v}_{1}(\theta) \sin \theta\right] \\
& +r^{\left(m^{\prime}-1\right)}\left[m^{\prime} w_{1}^{\prime}(\theta) \cos \theta-\dot{w}_{1}^{\prime}(\theta) \sin \theta\right]+\left[w_{1}^{\prime \prime}(\theta) \cos \theta-\dot{w}_{1}^{\prime \prime}(\theta) \sin \theta\right]+o(1), \\
& \frac{T_{\mathbb{R}_{21}}}{2 a}=r^{(m-1)}\left[m v_{2}(\theta) \cos \theta-\dot{v}_{2}(\theta) \sin \theta\right] \\
& +r^{\left(m^{\prime}-1\right)}\left[m^{\prime} w_{2}^{\prime}(\theta) \cos \theta-\dot{w}_{2}^{\prime}(\theta) \sin \theta\right]+\left[w_{2}^{\prime \prime}(\theta) \cos \theta-\dot{w}_{2}^{\prime \prime}(\theta) \sin \theta\right]+o(1), \\
& \frac{T_{\mathbb{R}_{12}}}{2 a}=r^{(m-1)}\left[m v_{1}(\theta) \sin \theta+\dot{v}_{1}(\theta) \cos \theta\right] \\
& +r^{\left(m^{\prime}-1\right)}\left[m^{\prime} w_{1}^{\prime}(\theta) \sin \theta+\dot{w}_{1}^{\prime}(\theta) \cos \theta\right]+\left[w_{1}^{\prime \prime}(\theta) \sin \theta+\dot{w}_{1}^{\prime \prime}(\theta) \cos \theta\right]+o(1), \\
& \frac{T_{\mathbb{R}_{22}}}{2 a}=r^{(m-1)}\left[m v_{2}(\theta) \sin \theta+\dot{v}_{2}(\theta) \cos \theta\right] \\
& +r^{\left(m^{\prime}-1\right)}\left[m^{\prime} w_{2}^{\prime}(\theta) \sin \theta+\dot{w}_{2}^{\prime}(\theta) \cos \theta\right]+\left[w_{2}^{\prime \prime}(\theta) \sin \theta+\dot{w}_{2}^{\prime \prime}(\theta) \cos \theta\right]+o(1) .
\end{aligned}
$$

From (5.1c) and (5.1d) it can be noted that, as required by the boundary condition (3.6), the free radial edge of the wedge $(\theta=\pi)$ is traction-free.

Expressing the deformation gradient in terms of polar coordinates, i.e.,

$$
[F]_{\alpha \beta}=\left[\begin{array}{ll}
\varphi_{1 . r} \cos \theta-\varphi_{1 . \theta} \frac{\sin \theta}{r} & \varphi_{1 . r} \sin \theta+\varphi_{1 . \theta} \frac{\cos \theta}{r} \\
\varphi_{2 . r} \cos \theta-\varphi_{2, \theta} \frac{\sin \theta}{r} & \varphi_{2, r} \sin \theta+\varphi_{2, \theta} \frac{\cos \theta}{r}
\end{array}\right],
$$


the components of the asymptotic Cauchy stress tensor can be obtained by the inverse Piola transform. This computation yields

$$
\begin{aligned}
& \frac{\delta T_{11}}{2 a}=r^{2(m-1)}\left[m^{2} v_{1}^{2}(\theta)+\dot{v}_{1}^{2}(\theta)\right]+2 r^{\left(m+m^{\prime}-2\right)}\left[m m^{\prime} v_{1}(\theta) w_{1}^{\prime}(\theta)+\dot{v}_{1}(\theta) \dot{w}_{1}^{\prime}(\theta)\right] \\
&+2 r^{(m-1)}\left[m v_{1}(\theta) w_{1}^{\prime \prime}(\theta)+\dot{v}_{1}(\theta) \dot{w}_{1}^{\prime \prime}(\theta)\right] \\
& \quad+r^{2\left(m^{\prime}-1\right)}\left[m^{\prime 2} w_{1}^{\prime 2}(\theta)+\dot{w}_{1}^{\prime 2}(\theta)\right]+2 r^{\left(m^{\prime}-1\right)}\left[m^{\prime} w_{1}^{\prime}(\theta) w_{1}^{\prime \prime}(\theta)\right. \\
&\left.\quad+\dot{w}_{1}^{\prime}(\theta) \dot{w}_{1}^{\prime \prime}(\theta)\right]+\left[w_{1}^{\prime \prime 2}(\theta)+\dot{w}_{1}^{\prime \prime 2}(\theta)\right]+o(1) \\
& \frac{\delta T_{12}}{2 a}=\frac{\delta T_{21}}{2 a}=r^{2(m-1)}\left[m^{2} v_{1}(\theta) v_{2}(\theta)+\dot{v}_{1}(\theta) \dot{v}_{2}(\theta)\right] \\
& \quad+r^{\left(m+m^{\prime}-2\right)}\left[m m^{\prime} v_{2}(\theta) w_{1}^{\prime}(\theta)+m m^{\prime} v_{1}(\theta) w_{2}^{\prime}(\theta)+\dot{v}_{1}(\theta) \dot{w}_{2}^{\prime}(\theta)+\dot{v}_{2}(\theta) \dot{w}_{1}^{\prime}(\theta)\right] \\
& \quad+r^{(m-1)}\left[m v_{1}(\theta) w_{2}^{\prime \prime}(\theta)+m v_{2}(\theta) w_{1}^{\prime \prime}(\theta)+\dot{v}_{1}(\theta) \dot{w}_{2}^{\prime \prime}(\theta)+\dot{v}_{2}(\theta) \dot{w}_{1}^{\prime \prime}(\theta)\right] \\
& \quad+r^{2\left(m^{\prime}-1\right)}\left[m^{\prime 2} w_{1}^{\prime}(\theta) w_{2}^{\prime}(\theta)+\dot{w}_{1}^{\prime}(\theta) \dot{w}_{2}^{\prime}(\theta)\right] \\
& \quad+r^{\left(m^{\prime}-1\right)}\left[m^{\prime} w_{1}^{\prime}(\theta) w_{2}^{\prime \prime}(\theta)+m^{\prime} w_{1}^{\prime \prime}(\theta) w_{2}^{\prime}(\theta)+\dot{w}_{1}^{\prime}(\theta) \dot{w}_{2}^{\prime \prime}(\theta)+\dot{w}_{1}^{\prime \prime}(\theta) \dot{w}_{2}^{\prime}(\theta)\right] \\
& \quad+\left[w_{1}^{\prime \prime}(\theta) w_{2}^{\prime \prime}(\theta)+\dot{w}_{1}^{\prime \prime}(\theta) \dot{w}_{2}^{\prime \prime}(\theta)\right]+o(1) \\
& \frac{\delta T_{22}}{2 a}=r^{2(m-1)}\left[m^{2} v_{2}^{2}(\theta)+\dot{v}_{2}^{2}(\theta)\right]+2 r^{\left(m+m^{\prime}-2\right)}\left[m m^{\prime} v_{2}(\theta) w_{2}^{\prime}(\theta)+\dot{v}_{2}(\theta) \dot{w}_{2}^{\prime}(\theta)\right] \\
& \quad+2 r^{(m-1)}\left[m v_{2}(\theta) w_{2}^{\prime \prime}(\theta)+\dot{v}_{2}(\theta) \dot{w}_{2}^{\prime \prime}(\theta)\right] \\
&+r^{2\left(m^{\prime}-1\right)}\left[m^{\prime 2} w_{2}^{\prime 2}(\theta)+\dot{w}_{2}^{\prime 2}(\theta)\right]+2 r^{\left(m^{\prime}-1\right)}\left[m^{\prime} w_{2}^{\prime}(\theta) w_{2}^{\prime \prime}(\theta)+\dot{w}_{2}^{\prime}(\theta) \dot{w}_{2}^{\prime \prime}(\theta)\right] \\
&+\left[w_{2}^{\prime \prime 2}(\theta)+\dot{w}_{2}^{\prime \prime 2}(\theta)\right]+o(1)
\end{aligned}
$$

where $\delta$ is specified by (4.26).

The above stress fields hold for $-\pi \leq-\bar{\theta}<-\pi / 2$. By vanishing in Eqs. (5.1a,b,c,d), $(5.2 \mathrm{a}, \mathrm{b}, \mathrm{c}, \mathrm{d})$, and $(5.4 \mathrm{a}, \mathrm{b}, \mathrm{c})$ the terms that contain the functions $w_{\alpha}^{\prime}(\theta)$ and $\dot{w}_{\alpha}^{\prime}(\theta)$, the expressions of the stress fields due to solution (4.23), which are valid for $0 \leq \bar{\theta}<\pi / 2$ and $-\pi / 2<-\bar{\theta} \leq 0$, are obtained.

For both solutions (4.23) and (4.24), the most singular term in the expressions of the Piola-Kirchhoff stresses has the order $O\left(r^{(m-1)}\right)$, where $m-1=(\pi-2 \phi) /(2 \phi)$, and becomes progressively more severe with increasing values of the wedge angle $\phi$, to reach the order $O\left(r^{-3 / 4}\right)$ for $\phi=2 \pi$ (case of the full-plane with a straight crack).

The most singular terms in the components of the Cauchy stress tensor are

$$
\begin{gathered}
\frac{\delta T_{11}}{2 a} \sim r^{2(m-1)}\left\{\frac{m^{2} A_{1}^{2}}{\cos ^{2} m \bar{\theta}}\right\}, \\
\frac{\delta T_{12}}{2 a}=\frac{\delta T_{21}}{2 a} \sim r^{2(m-1)}\left\{\frac{m^{2} A_{1} A_{2}}{\cos ^{2} m \bar{\theta}}\right\}, \\
\frac{\delta T_{22}}{2 a} \sim r^{2(m-1)}\left\{\frac{m^{2} A_{2}^{2}}{\cos ^{2} m \bar{\theta}}\right\} .
\end{gathered}
$$

To investigate the dominant character of the Cauchy stresses, when these are referred to the spatial coordinates, we introduce the spatial radius

$$
\rho(r, \theta)=\left(\varphi_{1}^{2}(r, \theta)+\varphi_{2}^{2}(r, \theta)\right)^{1 / 2} .
$$


Therefore, by considering only the most important terms in the deformation field, for $\theta \neq \bar{\theta}$, one obtains

$$
\rho(r, \theta) \sim \frac{A}{\cos m \bar{\theta}} r^{m}|\sin m(\theta-\bar{\theta})|, \quad \text { with } A=\left(A_{1}^{2}+A_{2}^{2}\right)^{1 / 2} .
$$

The order of singularity of the in-plane asymptotic components of the Cauchy stress tensor, which is stronger than that of the components of the Piola-Kirchhoff stress tensor, can then be expressed in the following two ways:

$$
T_{\alpha \beta} \sim O\left(r^{\frac{\pi-2 \phi}{\phi}}\right)=O\left(\rho^{\frac{2}{\pi}(\pi-2 \phi)}\right) .
$$

6. The particular case of a half-plane. The wedge model studied in the foregoing sections reproduces for $\bar{\theta}=0$ an interfacial crack problem between a sheet of compressible neo-Hookean material and a rigid substrate (Fig. 4). This case does not constitute a special limit case of a bimaterial crack problem, primarily because the matching conditions along the interface do not revert to the boundary conditions for this special problem.

Solution (4.23), specialized to the case $\bar{\theta}=0$, provides

$$
\begin{aligned}
& \varphi_{1}(r, \theta)=r^{1 / 2} A_{1} \sin \frac{\theta}{2}+r \cos \theta+o(r), \\
& \varphi_{2}(r, \theta)=r^{1 / 2} A_{2} \sin \frac{\theta}{2}+o(r) .
\end{aligned}
$$

To better understand the structure of the asymptotic deformation field (6.1a,b), we profit by a beautiful idea of Stephenson [39]. By introducing a special rigid-body rotation

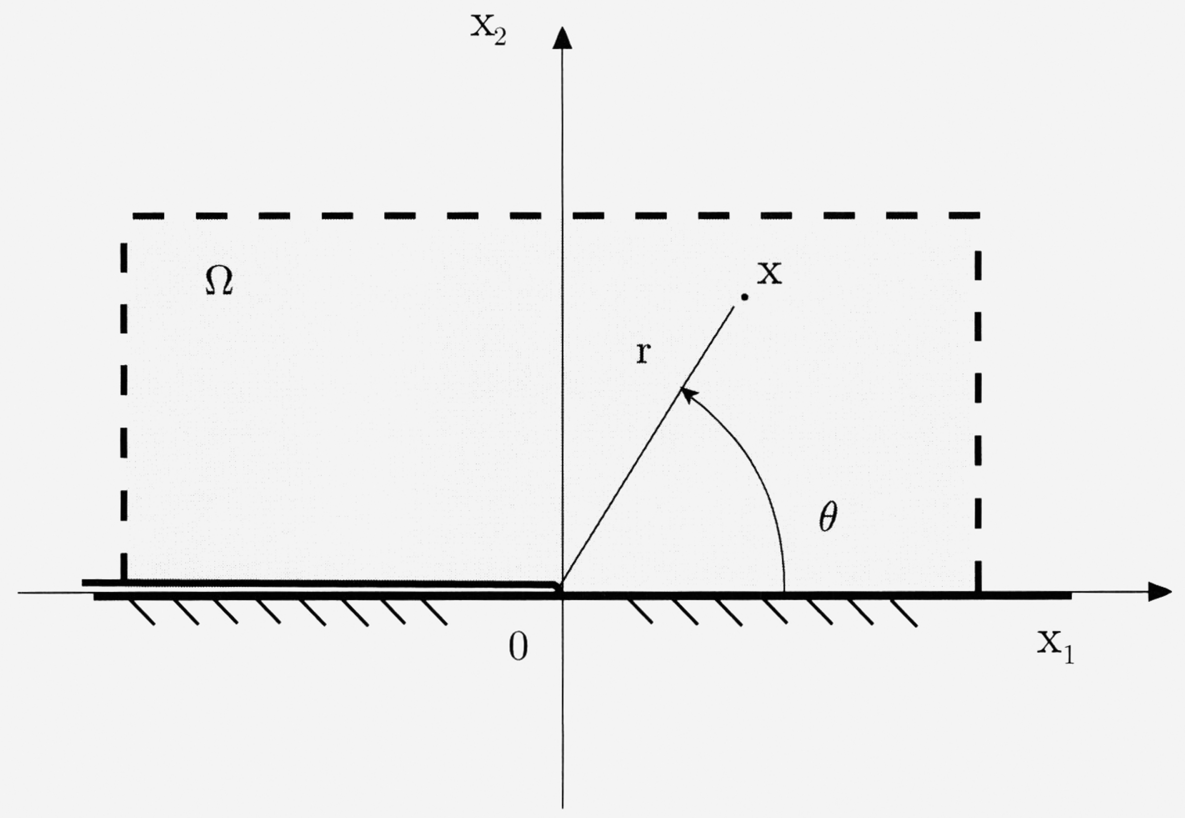

FIG. 4. Interface crack model 
$\mathrm{Q}^{\mathrm{T}} \in \mathrm{Orth}_{2}^{+}$such that

$$
\varphi^{*}=\mathrm{Q}^{\mathrm{T}} \varphi
$$

where $\mathbf{Q}^{\mathrm{T}}$ has the following components:

$$
\left[Q^{\mathrm{T}}\right]_{\alpha \beta}=\left[\begin{array}{cc}
\frac{A_{2}}{A} & -\frac{A_{1}}{A} \\
\frac{A_{1}}{A} & \frac{A_{2}}{A}
\end{array}\right],
$$

that correspond to a rotation angle given by $\tan \zeta=-\frac{A_{1}}{A_{2}}$, we obtain the canonical field

$$
\begin{aligned}
& \varphi_{1}^{*}(r, \theta)=\frac{A_{2}}{A} r \cos \theta+o(r), \\
& \varphi_{2}^{*}(r, \theta)=A r^{1 / 2} \sin \frac{\theta}{2}+\frac{A_{1}}{A} r \cos \theta+o(r) .
\end{aligned}
$$

The importance of (6.2) resides in the fact that, if only the dominant-order terms are taken into account, the rotation $\mathbf{Q}^{\mathrm{T}}$ permits obtaining the particular deformation field that satisfies the symmetry properties due to a mode I loading. This leads to the surprising result that, for arbitrarily given loading conditions at the infinite edge, the asymptotic deformation field is obtained by a mere rigid rotation of the canonical asymptotic field, or, likewise, of the solution of the mode I crack problem. Consequently, the nonlinear interface crack problem considered in this section cannot admit an antisymmetric solution. ${ }^{8}$

Equations $(6.3 \mathrm{a}, \mathrm{b})$ coincide formally with the solution recently proposed by Geubelle and Knauss for an incompressible generalized neo-Hookean material (cf. Eqs. (4.6) and (4.19) of [25], setting $n=1$ ).

The components of the Piola-Kirchhoff and Cauchy stress tensors, associated to the canonical deformation field $(6.3 \mathrm{a}, \mathrm{b})$, are

$$
\begin{aligned}
\frac{T_{\mathbb{E}_{11}}}{2 a}=\frac{A_{2}}{A}+o(1), & \frac{T_{\mathbb{R}_{12}}}{2 a}=o(1), \\
\frac{T_{\mathbb{R}_{21}}}{2 a}=-\frac{1}{2} A r^{-1 / 2} \sin \frac{\theta}{2}+\frac{A_{1}}{A}+o(1), & \frac{T_{\mathbb{R}_{22}}}{2 a}=\frac{1}{2} A r^{-1 / 2} \cos \frac{\theta}{2}+o(1),
\end{aligned}
$$

and

$$
\begin{gathered}
\frac{\delta T_{11}}{2 a}=\left(\frac{A_{2}}{A}\right)^{2}+o(1) \\
\frac{\delta T_{12}}{2 a}=\frac{\delta T_{21}}{2 a}=-\frac{1}{2} A_{2} r^{-1 / 2} \sin \frac{\theta}{2}+\frac{A_{1} A_{2}}{A^{2}}+o(1) \\
\frac{\delta T_{22}}{2 a}=\frac{1}{4} A^{2} r^{-1}-A_{1} r^{-1 / 2} \sin \frac{\theta}{2}+\left(\frac{A_{1}}{A}\right)^{2}+o(1) .
\end{gathered}
$$

With the use of $(6.3 \mathrm{a}, \mathrm{b})$, the spatial radial coordinate, evaluated along the line $\theta=0$, becomes $(\theta=\bar{\theta})$

$$
\rho(r, 0)=r
$$

It follows that the most singular component of the Cauchy stress tensor, $T_{22}$, has the asymptotic order $O\left(\rho^{-1}\right)$, which is stronger than the inverse square root singularity predicted by linear fracture mechanics.

\footnotetext{
${ }^{8} \mathrm{~A}$ similar result holds in many other contexts (see, for example, [23], [21], and [40]).
} 


\section{REFERENCES}

[1] M. L. Williams, Stress singularities resulting from various boundary conditions in angular corners of plates in extension, J. Appl. Mech. 19, 526-528 (1952)

[2] C. J. Tranter, Integral Transforms in Mathematical Physics, 2nd edition, Wiley, 1956

[3] E. Sternberg and W. Koiter, The wedge under a concentrated couple: A paradox in the twodimensional theory of elasticity, J. Appl. Mech. 25, 575-581 (1958)

[4] D. B. Bogy and E. Sternberg, The effect of couple-stresses on the corner singularity due to an asymmetric shear loading, Internat. J. Solids and Structures 4, 159-171 (1968)

[5] D. B. Bogy, On the problem of edge-bonded elastic quarter-planes loaded at the boundary, Internat. J. Solids and Structures 6, 1287-1313 (1970)

[6] D. B. Bogy, On the plane elastostatic problem of a loaded crack terminating at a material interface, J. Appl. Mech. 38, 911-918 (1971)

[7] A. E. Green and W. Zerna, Theoretical elasticity, Oxford, 1954

[8] M. Hetényi, A method of solution for the elastic quarter-plane, J. Appl. Mech. 27, 289-296 (1960)

[9] I. Babuska, K. Rektorys, and F. Vycichlo, Mathematische Elastizitätstheorie der ebenen Probleme, Akademie-Verlag, Berlin, 1960

[10] D. B. Bogy, Edge-bonded dissimilar orthogonal elastic wedges under normal and shear loading, J. Appl. Mech. 35, 460-466 (1968)

[11] J. Dundurs, Discussion of edge-bonded dissimilar orthogonal elastic wedges under normal and shear loading, J. Appl. Mech. 36, 650-652 (1969)

[12] D. B. Bogy and K. C. Wang, Stress singularities at interface corners in bonded dissimilar isotropic elastic materials, Internat. J. Solids and Structures 7, 993-1005 (1971)

[13] V. Hein and F. Erdogan, Stress singularities in a two-material wedge, Internat. J. Fract. Mech. 7, 317-330 (1971)

[14] J. Dundurs and M. S. Lec, Stress concentrations at a sharp edge in contact problems, J. of Elasticity 2, 109-112 (1972)

[15] E. E. Gdoutos and P. S. Theocaris, Stress concentrations at the apex of a plane indenter acting on an elastic half plane, J. Appl. Mech. 42, 688-692 (1975)

[16] P. S. Theocaris and E. E. Gdoutos, Stress singularities at vertices of composite plates with smooth or rough interfaces, Arch. Mech. Stosow. 28, 693-704 (1976)

[17] A. K. Rao, Stress concentrations and singularities at interface corners, Z. angew. Math. Mech. 51, 395-406 (1971)

[18] J. P. Dempsey and G. B. Sinclair, On the stress singularities in the plane elasticity of the composite wedge, J. of Elasticity 9, 373-391 (1979)

[19] J. P. Dempsey and G. B. Sinclair, On the singular behavior at the vertex of a bimaterial wedge, J. of Elasticity 11, 317--327 (1981)

[20] D. B. Bogy, The plane solution for anisotropic elastic wedges under normal and shear loading, J. Appl. Mech. 39, 1103-1109 (1972)

[21] A. M. Tarantino, The singular equilibrium field at the notch-tip of a compressible material in finite elastostatics, Z. angew. Math. Phys. 48, 370-388 (1997)

[22] F. S. Wong and R. T. Shield, Large plane deformations of thin elastic sheets of Neo-Hookean material, Z. angew. Math. Phys. 20, $176-199$ (1969)

[23] J. K. Knowles and E. Sternberg, Large deformations near a tip of an interface-crack between two Neo-Hookean sheets, J. of Elasticity 13, 257-293 (1983)

[24] P. H. Geubelle and W. G. Knauss, Finite strains at the tip of a crack in a sheet of hyperelastic material: I. Homogeneous case, J. of Elasticity 35, 61 98 (1994)

[25] P. H. Geubelle and W. G. Knauss, Finite strains at the tip of a crack in a sheet of hyperelastic material: II. Special bimaterial cases, J. of Elasticity 35, 99-138 (1994)

[26] P. H. Geubelle and W. G. Knauss, Finite strains at the tip of a crack in a sheet of hyperelastic material: III. General bimaterial case, J. of Elasticity 35, 139-174 (1994)

[27] A. M. Tarantino, Thin hyperelastic sheets of compressible material: field equations, Airy stress function and an application in fracture mechanics, J. of Elasticity 44, 37-59 (1996)

[28] J. E. Adkins, A. E. Green, and G. C. Nicholas, Two-dimensional theory of elasticity for finite deformation, Philos. Trans. Roy. Soc. A247, 279 306 (1954)

[29] J. E. Adkins and A. E. Green, Plane problems in second-order elasticity theory, Philos. Trans. Roy. Soc. A239, 557-576 (1957) 
[30] A. E. Green and J. E. Adkins, Large elastic deformation and nonlinear continuum mechanics, Oxford University Press, Oxford, 1960

[31] W. H. Yang, Stress concentration in a rubber sheet under axially symmetric stretching, J. Appl. Mech. 34, 942-946 (1967)

[32] A. E. H. Love, A Treatise on the Mathematical Theory of Elasticity, Cambridge University Press, Cambridge (1927) (reprinted by Dover Publications, New York, 1944)

[33] P. G. Ciarlet, Mathematical Elasticity. Vol. I: Three-Dimensional Elasticity, North-Holland, 1988

[34] S. S. Antman, Existence of solutions of the equilibrium equations for nonlinearly elastic rings and arches, Indiana University Mathematics Journal 20, 281-302 (1970)

[35] S. S. Antman, Regular and singular problems for large elastic deformations of tubes, wedges, and cylinders, Arch. Rational Mech. Anal. 83, 1-52 (1983)

[36] P. Podio Guidugli and G. Vergara Caffarelli, Extreme elastic deformations, Arch. Rational Mech. Anal. 115, 311-328 (1991)

[37] P. G. Ciarlet and G. Geymonat, Sur les lois de comportement en élasticité non-linéaire compressible, C. R. Acad. Sci. Paris Sér. II 295, 423-426 (1982)

[38] K. Ch. Le, On the singular elastostatic field induced by a crack in a Hadamard material, Quart. J. Mech. Appl. Math. 45, 101-117 (1992)

[39] R. A. Stephenson, The equilibrium field near the tip of a crack for finite plane strain of incompressible elastic materials, J. of Elasticity 12, 65-99 (1982)

[40] K. Ch. Le and H. Stumpf, The singular elastostatic field due to a crack in rubberlike materials, J. of Elasticity 32, 183-222 (1993) 